\title{
Doğu Anadolu'daki Obsidiyen Kaynak Alanlarının Belirlenmesinde Jeomorfolojik ve Volkanolojik Göstergelerin Önemi
}

\author{
The Importance of the Geomorphological and Volcanological Indicators in Determining \\ Obsidian Source Areas
}

\section{Ebru AKKÖPRÜ1, Damase MOURALIS ${ }^{2}$, Anne-Kyria ROBIN ${ }^{3}$ Catherine KUZUCUOĞLU ${ }^{3}$ ve M. Korhan ERTURAÇ ${ }^{4}$}

${ }^{\text {I} Y u ̈ z u ̈ n c u ̈ ~ Y ı l ~ U ̈ n i v e r s i t e s i, ~ E d e b i y a t ~ F a k u ̈ l t e s i, ~ C o g ̆ r a f y a ~ B o ̈ l u ̈ m u ̈, ~} 65040$ Van (ebruakkopru@yahoo.com)

${ }^{2}$ Université de Rouen \& CNRS (Laboratoire IDEES, UMR 6266). Rue Lavoisier, 76831 Mont-Saint-Aignan (France)

${ }^{3}$ Université Paris 1 Panthéon-Sorbonne, \& CNRS (LGP, UMR 8591).

1 Place Aristide Briand, 92195 Meudon cedex (France), ${ }^{4}$ Sakarya Üniversitesi, Coğrafya Bölümü, 54187 Sakarya

\section{$\ddot{O z Z}$}

Arkeolojik kazı alanlarında bulunan el aletlerinden anlaşıldığı üzere Doğu Anadolu Bölgesi volkanik alanlarına ait obsidiyenler tarih öncesi dönemlerde ticareti yapılarak çok geniş alanlara yayılmıştır. Bu nedenle Doğu Anadolu Bölgesi’nde obsidiyenin yayılımı ve volkanik-jeomorfolojik özellikleri ile alakalı bir çalışma yapmak önemlidir.

Bu çalışmada, Doğu Anadolu Bölgesi volkanik alanlarında jeomorfolojik ve jeolojik yaklaşımla yapılan arazi çalışmalarından örnekler verilerek obsidiyen kaynaklarının belirlenmesinde volkanolojik ve jeomorfolojik göstergelerin öneminden bahsedilmiştir.

Obsidiyenin temel özellikleri (renk, doku, dayanıklılık, sertlik vb), hammadde olarak varlığı, miktarı ve ulaşılabilirliği volkanik ve jeomorfolojik süreçler ile yakından ilişkilidir. Örneğin; obsidiyenin kimsayal ve fiziksel özellikleri büyük oranda volkanizmanın kontrolü altında belirmektedir. Obsidiyenin yüzlek alanlarının yayılımında ve oluşum tiplerinde (dom, dayk, akma, vb) magmatik süreçler önemli rol oynar. Volkanik bir masif içindeki obsidiyenin bulunması volkanizma sonucudur ancak daha sonra aynı alan içinde varlığını sürdürmesi tamamen aşınma süreçlerinin türüne ve şiddetine de bağlıdır. Volkanik 
topografyanın evrimi obsidiyenin hammadde olarak bulunup işlenip kullanılmasında önemlidir. Şöyle ki volkanik alanda oluşan sonraki volkanik faaliyetler ve erozyon süreçleri obsidiyenin fosilleşmesine veya tamamen aşınıp tahrip olmasına neden olabilir. Ayrıca bu çeşit bir etki ikincil kaynakları olarak bilinen kolüvyal veya alüvyal malzeme içindeki obsidiyen varlığını ve oranını da değiştirir.

Anahtar Kelimeler: Doğu Anadolu, Volkanizma, Obsidiyen, Jeomorfoloji,

\section{ABSTRACT}

As understood from the artifacts found in the archaeological excavation areas, the obsidian belonging to the volcanic areas of the Eastern Anatolian Region spread to a very wide area by being traded in prehistoric times. For this reason, it is important to carry out a study on obsidian diffusion and volcanicgeomorphological features in the Eastern Anatolia Region.

In this study, the importance of volcanological and geomorphological indicators in the determination of obsidian sources is given by examplifying field studies with geomorphological and geological approaches in the volcanic areas of Eastern Anatolia Region

The basic characteristics of obsidian (color, texture, durability, hardness, etc.) presence, quantity and availability as raw materials are closely related with volcanic and geomorphological processes. For example; The chemical and physical properties of the obsidian appear largely under the control of volcanism. Magmatic processes play an important role in the spread of the obsidian surface areas and in the formation types (dom, dyke, flow, etc.). The presence of obsidian in a volcanic massif is the result of volcanism, but at the same time, its continuation in the same area later also depends on the type and severity of the entire erosion process. Namely, the next volcanic activity and or erosional processes in the volcanic area may cause the obsidian to fossilize or be completely eroded and destroyed. In addition, this kind of effect also changes the presence and proportion of obsidian in the colluvial and alluvial material known as secondary sources on the area.

Keywords: Eastern Anatolia, Volcanism, Obsidian, Geomorphology

\section{GíRiş}

Türkiye'nin volkan topografyası bakımından en geniş yayılım alanına sahip bölgesi Doğu Anadolu'dur ve obsidiyen varlığı bakımından da oldukça zengindir. Bölge aynı zamanda zengin bir arkeolojik geçmişe de sahiptir. Kolay işlenebilen pek çok materyal gibi obsidiyen de tarihin ilk dönemlerinde çeşitli el aletleri ve malzeme yapımında yaygın bir şekilde kullanılmıştır. $\mathrm{Bu}$ açıdan bakıldığında bölgenin obsidiyen ana kaynakları açısından ve ayrıca bölgede bulunan göl-akarsu taraçaları ve ova dolgusu içerisinde obsidiyen varlığ 1 ve aynı zamanda kültürel katlarda bulunan malzeme açısından önemi büyüktür.

Geobs projesi çerçevesinde (http://geobs. univ-rouen.fr) Doğu Anadolu Bölgesi’ndeki 
obsidiyen yüzeylenmelerine yönelik kapsamlı bir çalışma yürütülmektedir. Bu makalede sunulan bilgilerve sonuçlar söz konusu proje çalışmalarının bir kısmını içermektedir. Anadolu'daki obsidiyen araştırmaları Cann ve Renfrew (1964) tarafından yapılan çalışmalar ile başlamış ve daha sonra sayısı giderek artmıştır (Cauvin vd. 1998; Chataigner vd. 1998; Poidevin, 1998; Özdoğan ve Başgelen, 1999). Aynı zamanda birçok arkeolojik kazı alanlarında bulunan ve kaynakları Orta Anadolu ve Kafkasya'daki volkanik alanlarla ilişkilendirilmiş obsidiyen eserler ortaya çıkarılmıştır (Cauvin vd.1998; Erturaç vd. 2010; Binder vd. 2011; Y1lmaz vd. 1998; Chataigner, 1994; Cauvin ve Chataigner, 1998; Astruc vd. 2007, Khalidi vd. 2009). Ancak birçok obsidiyen el aletinin volkanik anlamda kökeni hala tam olarak bilinmemektedir. $\mathrm{Bu}$ nedenle Geobs programı kapsamında Doğu Anadolu'daki farklı volkanik alanlara ait obsidiyenlerin esas kaynak alanları belirlenerek haritalanmış ve aynı zamanda farklı yöntemler kullanarak hem arkeolojik eserlerin ve hem de araziden toplanan obsidiyen örneklerinin jeokimyasal analizler yapılmıştır. Tüm sonuçlar bir veri tabanı yardımıyla sistematik bir şekilde düzenlenmektedir. Böylece obsidiyenin mekânsal dağılımı disiplinler arası bir yaklaşımla ( jeoloji, arkeoloji jeomorfoloji ve arkeometri) açıklanmaya çalışılacaktır. 2014 yılında başlayan çalışmalar henüz tamamlanmamış olup ancak elde edilen sonuçların bir kısmı yayınlanmıştır (Robin vd. 2016).

\section{DOĞU ANADOLU BÖLGESINDEKI OBSIDIIYEN KAYNAK ALANLARI}

Geobs programı çerçevesinde Doğu Anadolu'da obsidiyen yayılımı bilinen 14 volkanik alan incelenmektedir (Şekil 1). Birincil kaynak alanı olarak belirlenmiş bu 14 volkanik alanı içinde aynı zamanda işlenmiş obsidiyenlerin varlığı bilinen tortul alanlardaki (flüvyal taraça dolguları, ova dolguları vb.) ikincil kaynak alanları da araştırma kapsamında incelenmektedir.

Van Gölü çevresinde dört volkanik alan içinde obsidiyen yayılımı olduğu bilinmektedir. Bunlar; Nemrut Volkanı (2935m), Süphan Volkanı (4434m), Meydan (3290m) ve Tendürek volkanik dağlarıdır. Nemrut volkanı son yıllarda yapılan çalışmalarda detaylı olarak incelenmiştir (Özdemir vd. 2006; Karaoğlu vd. 2005, Robin vd 2016). Meydan dağ1 ve çevresindeki obsidiyenli riyolitik dom ve dayklarının olduğu bilinmektedir (Aslan 1994; Astruc vd. 2007; Khalidi vd. 2009). Yayınlanmış çalışmalar içinde Süphan (Oddone vd. 1997; Bigazzi vd.1997; Poidevin 1998) ve Tendürek (Pearce vd., 1990; Blackman vd., 1998) volkanlarına ait obsidiyen yayılımı ile ilgili bilgiler bulunmaktadır. Bingöl ve civarında Poidvein (1998) tarafından yapılan çalışmada dört obsidiyen deposu belirlenerek yaşlandırma çalışmaları yapılmıştır. Muş ve çevresindeki obsidiyenler hakkında bilgi çok azdır (Bigazzi vd., 1998, Oddone vd., 1997). Erzincan obsidiyenlerine yönelik bazı kimyasal analiz sonuçları Keller ve Seifried (1990) ile Poidevin (1998) tarafindan yayınlanmıştır. 


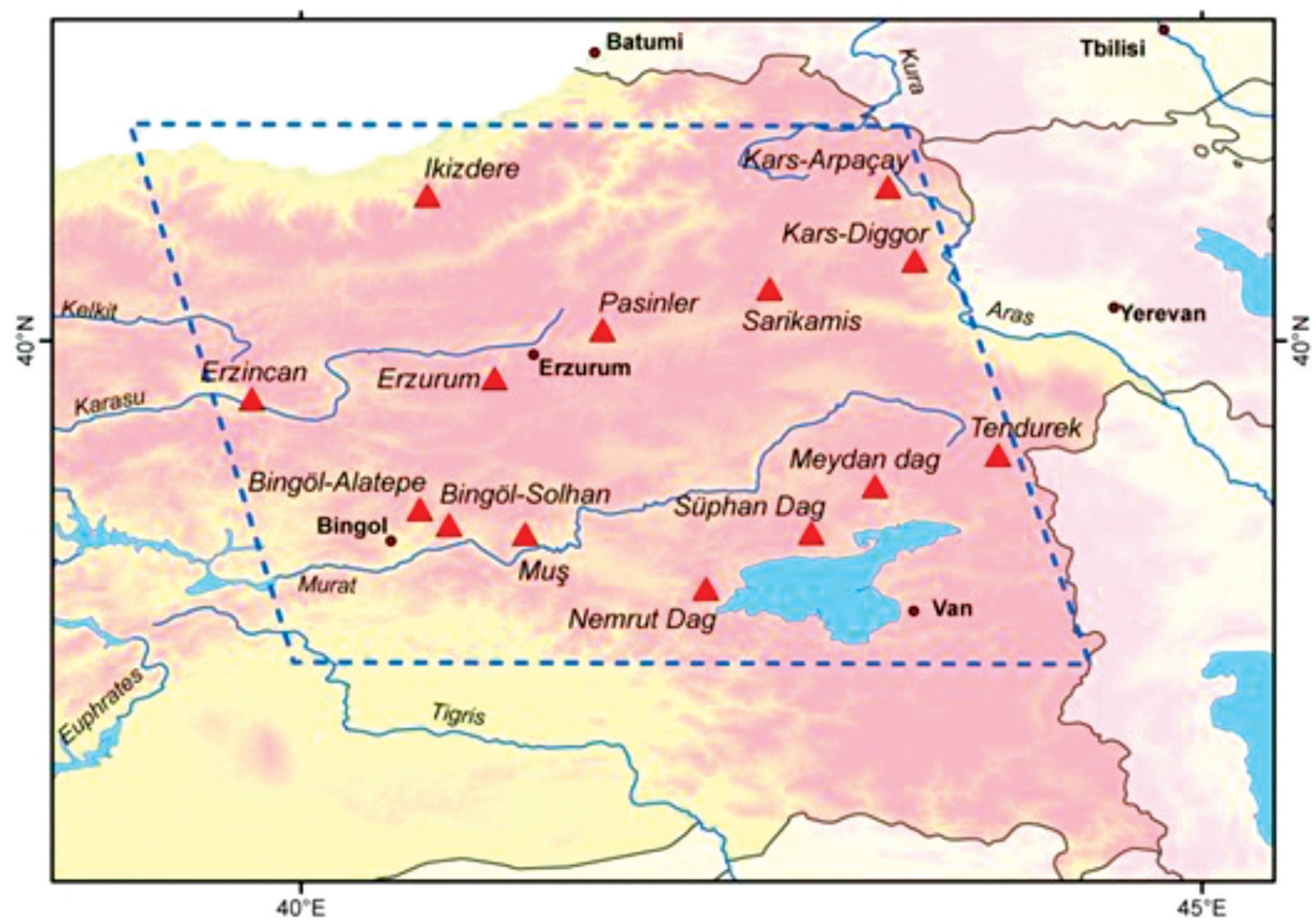

Şekil 1. Doğu Anadolu Bölgesi obsidiyen kaynak alanlarını gösterir harita

Figure 1. Map showing obsidian resource areas in Eastern Anatolia region

Daha doğuda Erzurum ve çevresinde Ercan vd. (1996) tarafindan farklı obsidiyen depoları tespit edilmiştir. Bunlardan bazılarının yaşlandırma analizleri (Biggazi vd.1997) ve diğer bazı örneklerin jeokimyasal analizleri yapılmıştır (Poidevin 1998). Erzurum-Pasinler çevresindeki obsidiyenler farkl1 jeolojik özelliklere sahiptirler ve obsidiyen domları ya piroklastikler ya da alüvyonlar içinde bulunmaktadır (Poidevin 1998). Sarıkamış'da iki obsidiyen kaynağında Keller ve Seifried (1990) tarafindan jeokimyasal analizler yapılmıştır. Kars civarındaki obsidiyen lokasyonları net bir şekilde belirlenerek üç ayrı alan olarak açıklanmıştır. Bunlar; Digor, Gelirli ve Arpaçay'dır (Ercan vd. 1996, Poidevin 1998) ve jeokimyasal analizleri Renfrew vd.(1996) ve Gratuze (1998) tarafından yayınlanmıştır. Son olarak Karadeniz Bölgesi'nde Trabzon yakınlarında İkizdere obsidiyenleri Yeğingil vd (2002) tarafından çalışılmıştır. Ayrıca Chataigner ve Gratuze (2013a ve 2013b) ve Chataigner vd. (2013) Doğu Anadolu ve özellikle Erzurum-Kars alanındaki obsidiyenler ile alakalı yeni veriler yayınlamışlardır.

\section{Kullanılan Yöntemler}

\section{Arazi Çalışmaları}

Araştırma kapsamında 2013-2015 yılları arasında Kars-Sarıkamış, Erzurum-Pasinler, Van Gölü 
çevresindeki, Muş, Bingöl-Solhan, BingölAlatepe volkanik alanlarında arazi çalışmaları yapılmıştır. Arazide, GPS ile yerleri kaydedilen örnek alımlarının yanı sıra, birincil ve ikincil kaynak alanlarının jeomorfolojik ortamı, stratigrafik durumu ve fasiyes yapısı araştırılmıştır. Obsidiyen örneklerinin tümü için daha önceden parametreleri belirlenmiş olan veri tabanı için bilgiler toplanmıştır. Tüm obsidiyen yüzlek alanlarında örneklenen obsidiyenlerin renk, parlaklık, yapı ve doku özellikleri tanımlanmıştır. Örnekler yaşlandırma analizleri ve jeokimyasal analizler için fiziksel özelliklerinin değişkenliği dikkate alınarak sistematik bir şekilde alınmıştır. Ayrıca daha önce farklı araştırmacılar (Biggazi vd. 1997; Frahm 2012) tarafindan yapılmış ve obsidiyen kaynak alanlarını gösteren haritalar güncellenerek yeni tespit edilen alanlar haritalar üzerinde GPS bilgileri kullanılarak işaretlenmiştir.

\section{Yaşlandırma Analizleri}

Obsidiyen yaşlandırma çalışmalarında iki farklı yöntem kullanılmaktadır: 1- Fizyon izi yaşlandırması (FTD) ve 2- K/Ar ve $\mathrm{Ar} / \mathrm{Ar}$ radyometrik yaşlandırma yöntemleri. Geobs projesi kapsamında ${ }^{40} \mathrm{Ar} /{ }^{39} \mathrm{Ar}$ yaşlandırma yöntemi kullanılmaktadır. Obsidiyen örneklerinin yaşlandırma analizleri Fransa'da LSCE (Laboratoire des Science du Climat et de L'Environnement) laboratuarlarında ve analiz için hazırlıklar Nomade vd. (2010) yayınladıkları prosedürlere uyularak yapılmaktadır. Toplamda 25 örnek yaşlandırma analizi yapılmak üzere alınmıştır. Bunlardan; 11 örnek Bingöl-Alatepe ve Bingöl- Solhan çevresindeki volkanik alanlarından 1 örnek Nemrut volkanı-Sicaksu obsidiyen kaynağından (A.K. vd. 2016), 4 örnek
Meydan-Gürgürbaba volkanik alanından, 3 örnek Kars-Sarıkamış obsidiyenlerinden, 6 örnek ise Erzurum- Pasinler ve Palendöken volkanik alanlarından alınmıştır. Yaşlandırma analizleri tamamlanmamış olup daha sonraki yayınlarda sonuçlar verilecektir.

\section{Jeokimyasal Analizler}

Obsidiyenlerin karakteristik özelliklerinin saptanmasinda yapılan jeokimyasal analizler Fransa-Orléans'da IRAMAT laboratuarında LA_ICP_MS (Mass Spektrometresi)yöntemi ve arazide portatif-XRF kullanılarak yapılmaktadır. Son yıllarda obsidiyen konulu yayınlarda özellikle p-XRF kullanıldığı gözlenmektedir. $\mathrm{Bu}$ yöntem portatif olma özelliğinden dolayı arazide ya da müzelerde analiz yapabilme performansına sahiptir. Böylece örneklerin laboratuar ortamına taşınmasına gerek yoktur ve analizler hızlı bir şekilde yapılabilmektedir. Özellikle üniversite ya da müze arşivlerinde bulunan obsidiyen arkeolojik eserlerinin, ortamlarından ayırmadan analizlerini yapmak mümkün olmaktadır. Arazi çalışmaları ve laboratuar analizleri henüz devam etmektedir. Bu çalışmada analizlerden elde edilen ilk sonuçlar paylaşılmıştır.

\section{JEOMORFOLOJIK VE VOLKANOLOJIKK GÖSTERGELER ILLE OBSIDIYYEN KAYNAK ALANLARININ BELIRLENMESI}

\section{Obsidiyenlerin Birincil ve İkincil Kaynak Alanları}

Doğu Anadolu Bölgesinde 2013-2015 y1lları arasında yapılan arazi çalışmaları sırasında öncelikle jeoloji haritalarında işaretlenmiş, obsidiyen varlığ 
ve lokalizasyon çalışmaları yapılmıştır. Aynı zamanda daha önce tespit edilmemiş obsidiyen yüzeylenmeleri de haritalar üzerine işaretlenmiştir. Haritalama çalışmalarının yanında volkanik alanların jeomorfolojik gelişimini anlamaya yönelik arazi çalışmaları yapılmıştır. Obsidiyen ana kaynak alanlarının dış etken ve süreçler ile nasıl bir etkileşimde olduğu ve volkanik yapının bu etkileşimle nasıl bir değişime uğradığı araştırılmıştır.

Obsidiyen kaynak alanları birincil ve ikincil kaynaklar olarak iki grupta incelenmiştir.
Birincil kaynaklar obsidiyenlerin oluştuğu ve yüzeylendiği alanlardır. Bu alanlar aynı volkan kompleksi içinde bir dayk, bir obsidiyen domu veya bir volkanik akış olabilir. İkincil kaynaklar ise işlenmiş obsidiyenlerin bulunduğu alanlardır. Buralar genellikle obsidiyenin diş etken ve süreçler ile aşınıp taşındığı ve biriktiği akarsu taraçaları, akarsu yatakları, vadiler veya arkeolojik kazı alanlarıdır. Bingöl ve çevresindeki volkanik alanlarda 2015 yılında yapılan arazi çalışmaları sırasında birincil ve ikincil kaynak alanları tespit edilmiştir (Şekil 2).

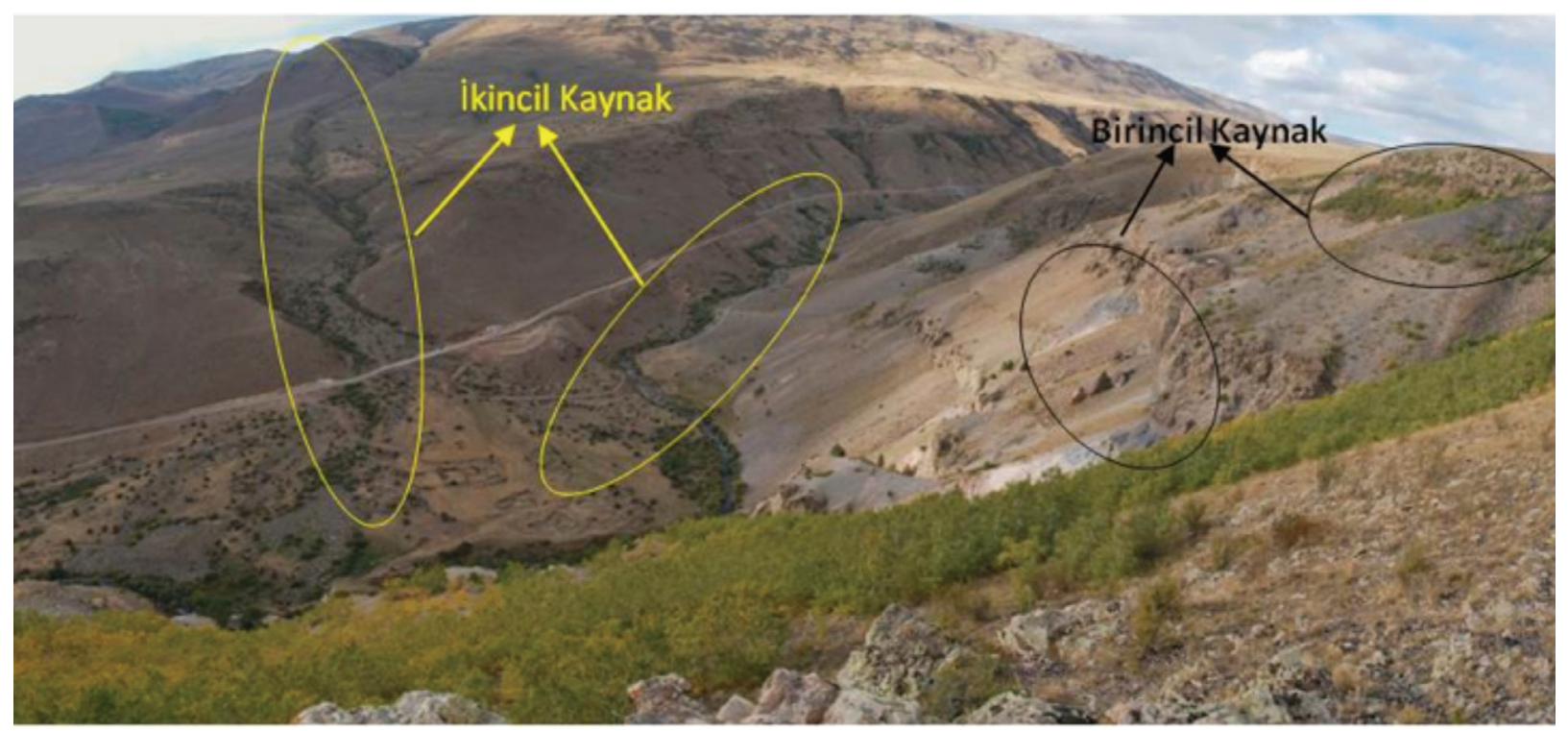

Şekil 2. Bingöl volkanik alanında Birincil ve İkincil Obsidiyen kaynak alanları

Figure 2. Primary and secondary obsidian sources in Bingöl volcanic area 
Bingöl ve çevresindeki volkanik alanlarda olduğu gibi obsidiyenler farklı konumlarda bulunabilmektedir. Örneğin obsidiyen karakterli riyolitik bir dom içinde olabileceği gibi piroklastik akış çökelleri içinde ikincil olarak da bulunabilmektedir. Bununla birlikte yüzeyde tüm bir tepe üzerinde yaygın ve işlenmiş halde (Şekil 3a-b) ya da Solhan ve çevresindeki gibi akarsu taraçaları içinde taşınmış, farklı renk ve dokuda obsidiyenler vardır (Şekil 4).

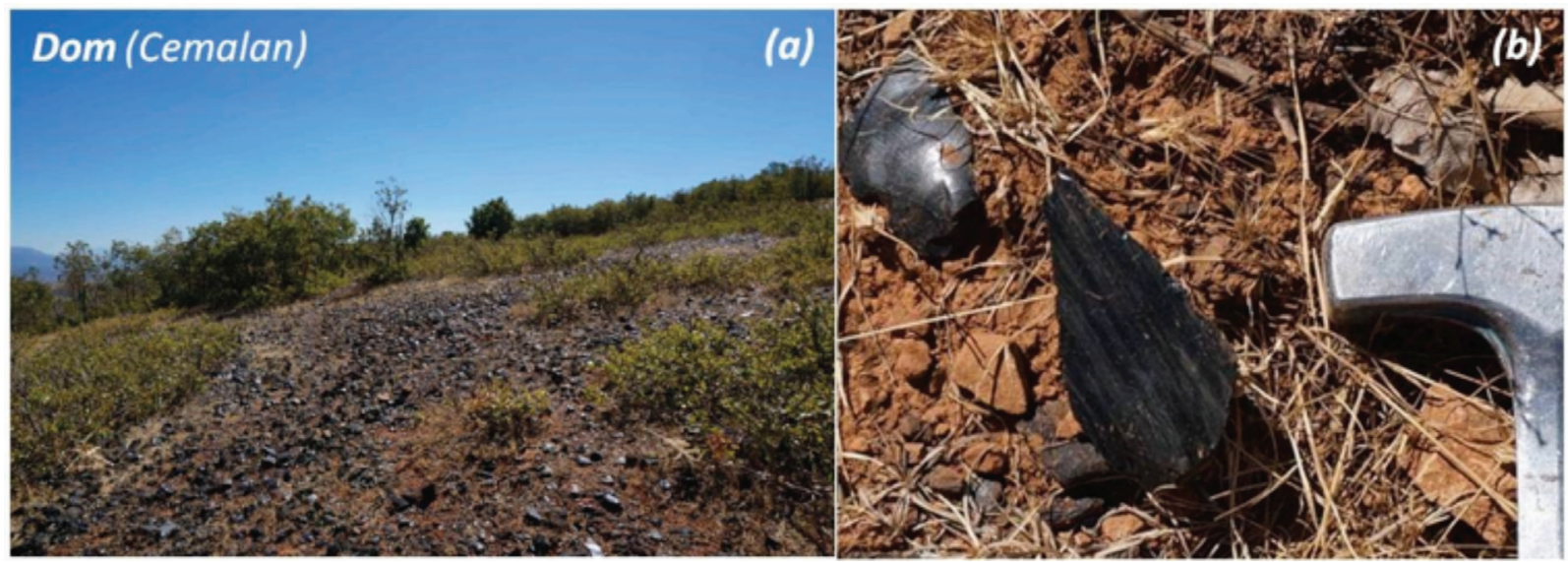

Şekil 3a-b. Bingöl- Cemalan çevresinde İşlenmiş Obsidiyenler

Figure 3a-b. Reworked Obsidian around Bingöl-Cemalan

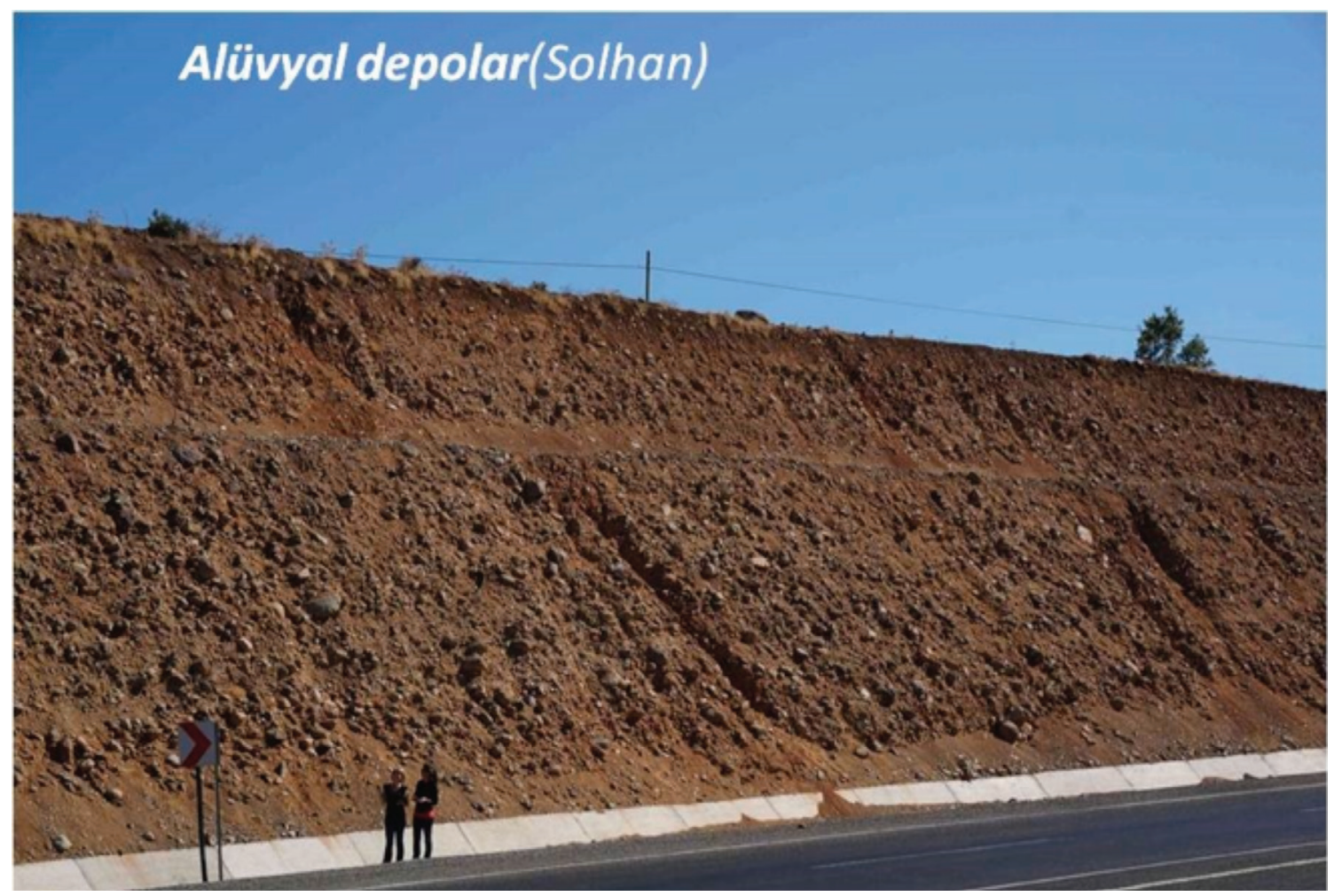

Şekil 4. Bingöl- Solhan civarındaki alüvyal depo içindeki obsidiyenler

Figure 4. Obsidian in the alluvial deposits around Bingöl-Solhan 
Birincil Kaynak Alanlarında Obsidiyenlerin Yüzeylenme Farklılıklart ve Fiziksel Özellikleri

Doğu Anadolu Bölgesi'nde aynı volkanik kompleks içindeki obsidiyenler farklı yüzeylenmeler gösterebilir. $\mathrm{Bu}$ yüzeylenmeler obsidiyenin oluştuğu ortam özelliklerinin yanı sıra sertlik,işlenebilirilik vb. fiziksel özellikleri hakkında da bilgiler vermektedir. Örneğin MaydanGürgürbaba volkanik alanı (Van Gölü Havzası) içinde yapılan arazi çalışmaları sırasında masif ve büyük hacimli kayalar halinde obsidiyenler görülürken (Şekil 5) Nemrut Volkanı çevresinde riyolit ya da ignimbiritler içinde nodüller halinde ve çizgisel formda obsidiyenler bulunmaktadır (Şekil 6a-b).

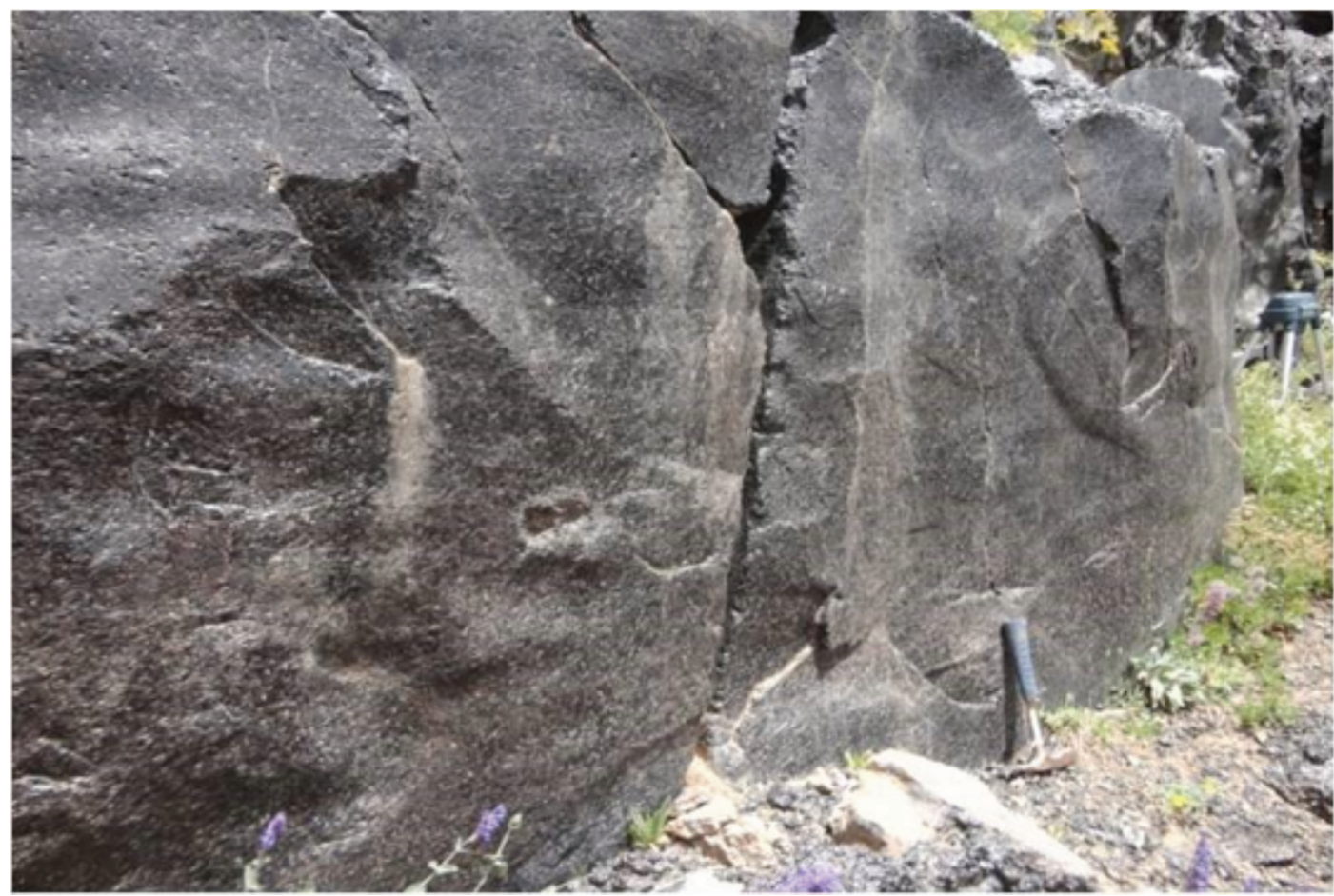

Şekil 5. Masif Kaya halinde Meydan-Gürgürbaba obsidiyenleri

Figure 5. Massive rock Obsidian of Meydan-Gürgürbaba

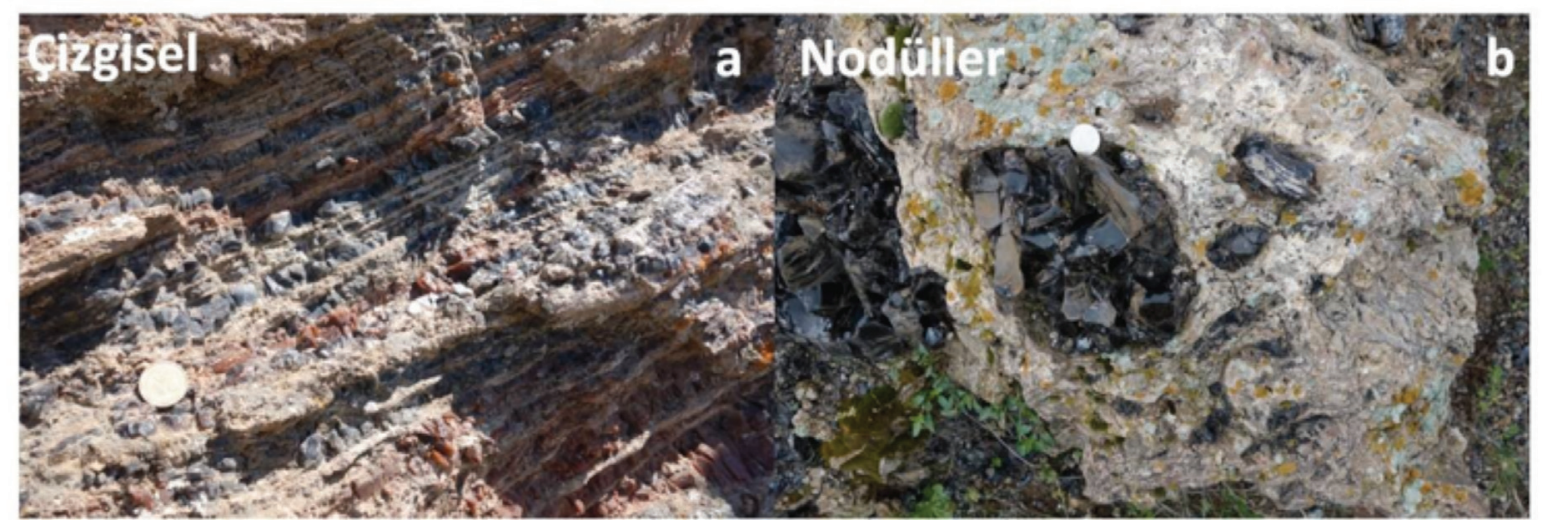

Şekil 6. Nodüler ve çizgisel formdaki Nemrut Volkanı Obsidiyenleri

Figure 6. In nodular and linear form obsidian of Nemrut volcano 


\section{Obsidiyen Kaynaklarının Volkanolojik Evrimi}

Geobs projesi kapsamında yürütülen çalışmalar obsidiyenlerin sadece oluşum, dağılım, fiziksel ve kimyasal özellikleri konusunda değil aynı zamanda obsidiyenlerin bulundukları ortamdaki volkanolojik ve jeomorfolojik özelliklerinin de doğru yorumlanmasına yönelik yapılmaktadır. Birincil kaynakların tespitinde volkanolojik gelişimi anlamak için aynı kompleks içinde aynı alandan ancak farklı fiziksel özelliklere sahip obsidiyenler üzerinde jeokimyasal analizler yapılmıştır. Böylece volkanın obsidiyen içerikli faaliyetleri sırasinda magmanın kimyasal özellikleri ve volkanolojik evrimi araştırılmıştır. Örneğin Şekil 7'de görüleceği üzere Nemrut volkanından alınan obsidiyenler mineral içeriklerine göre gruplanmalar gösterir. $\mathrm{Bu}$ gruplanmalardan Nemrut volkanının oluşum ve gelişim evrelerinde magmanın nasıl bir mineralojik değişim gösterdiği ve obsidiyenleri farklı fazlarda oluştuğu anlaşılmaktadır.

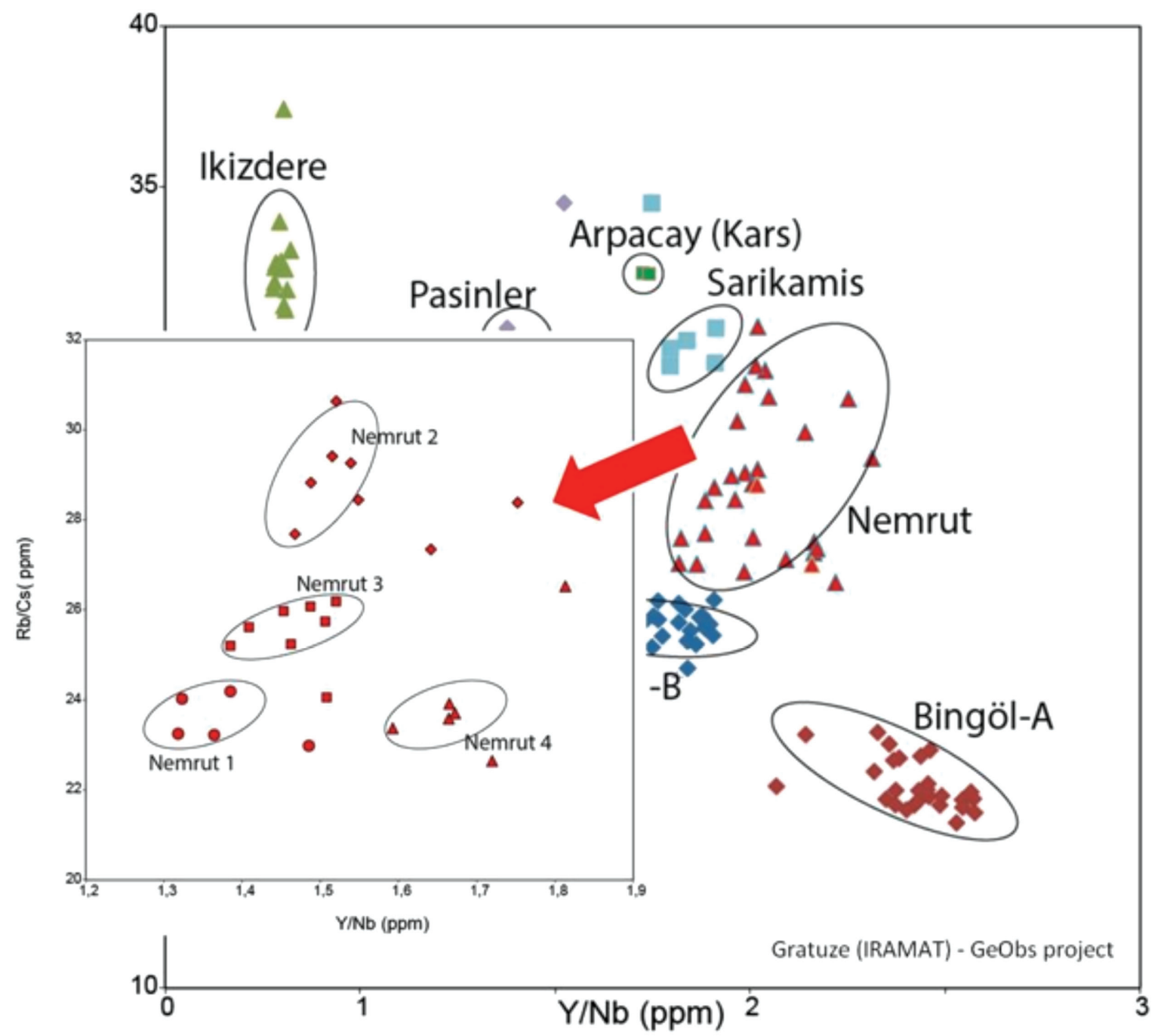

Şekil 7. Nemrut Obsidiyenlerinin jeokimyasal analiz sonuçları

Figure 7. Geochemical analysis results of Nemrut obsidian 


\section{SONUÇ VE TARTIŞMA}

Doğu Anadolu Bölgesi Obsidiyenlerinin araştırıldığ1 Geobs projesi kapsamında 20132015 yılları arasında volkanik alanlar içinde kapsamlı arazi çalışmaları yapılarak obsidiyen kaynak alanları tespit edilmiştir. Obsidiyen kaynakları iki gruba ayrılarak (birincil ve ikincil kaynaklar) bulundukları konumların volkanolojik ve jeomorfolojik özellikleri araştırılmıştır. Birincil kaynak alanlarının oluşumu volkanizma kontrolü altında gelişirken ikincil kaynak alanları daha çok volkanik alanın jeomorfolojik evrimi ile bağlantılı olarak oluşmuşlardır. Aynı volkanik alan içinde birbirine çok yakın hatta bazı yerlerde iç içe bulunan, ancak fiziksel özellikleri, yüzeylenme farklılıkları bakımından birbirinden ayrılan obsidiyenler bulunmaktadır. $\mathrm{Bu}$ obsidiyenlerin ayrımını yapmak ancak detaylı ve kapsamlı arazi çalışmalarının yapılmasıyla mümkün olmuştur. Farklı obsidiyenlerin jeokimyasal analizleri ile volkanın obsidiyen içerikli malzeme yaydıkları fazlardaki mineralojik özellikleri ile volkanın evrimini ve yaşlandırma analizleri ile de volkanın kronolojisini anlamaya yönelik çalışmalar yürütülmektedir.

Geobs projesi devam eden bir projedir (http://geobs.univ-rouen.fr).

Obsidiyen örneklerinin jeokimyasal ve yaşlandırma analizleri henüz tamamlanmamış olup sadece Nemrut volkanına ait iki kaynak alanında yapılan çalışmaların sonuçları Robin vd.(2016) tarafından yayınlanmıştır.

\section{EXTENDED SUMMARY}

The region which has the widest spreading area in terms of volcanic topography of Turkey is Eastern Anatolia and is also rich in obsidian existence. The region also has a rich archaeological background. As understood from the artifacts found in the archaeological excavation areas, the obsidian belonging to the volcanic areas of the Eastern Anatolian Region spread to a very wide area by being traded in prehistoric times.

In the framework of the Geobs project (http://geobs.univ-rouen.fr) a comprehensive study is carried out on obsidian exposures in Eastern Anatolia. The information and some results presented in this article include a part of the project work.

14 volcanic areas(Van Lake Basin, Erzurum- Kars Area, Muş, Bingöl, Erzincan) in Eastern Anatolia where obsidian propagation is observed are examine within the scope of the study. In the field studies obsidian samples the location pointsof which recorded by GPS are collected, at the same time the geomorpgological environment, stratigraphical status and facies structure of the primary and secondary source areas are investigated, data and informations are recorded. The primary sources are the areas where obsidian is existed and exposed. These areas may be a dike, an obsidian-containing rhyolite domes or a volcanic flow within the same volcano complex. Secondary sources are processed obsidian areas. These are usually river terraces, river beds, valleys or archeological excavation areas where the obsidian erodes and accumulates with external factors and processes.

For all sampled obsidian; the color, brightness and texture properties has been defined; in all obsidian surface areas. The samples were taken systematically for dating analyzes and geochemical analyzes, taking into account the variability of physical properties. In addition, 
maps that were previously made by different researchers (Biggazi et al., 1997; Frahm 2012) and showing obsidian source areas were updated and newly detected areas were marked on the maps using GPS information Alongside mapping studies, field studies were carried out to determine the geomorphological development of volcanic areas.It has been researched how the obsidian main resource areas interact with external factors and processes and how the volcanic structure is changing with this interaction.

The obsidian can show different outcrops in the same volcanic complex. These extras can give information about the environmental properties of the obsidian as well as the physical properties such as hardness, machinability etc. Laboratory studies have been carried out to comprehened volcanological evolution. In the same volcanic complex, obsidian with different physical properties were analyzed geochemically.

The Geobs project is an ongoing project (http://geobs.univ-rouen.fr). The geochemical and dating analyzes of the obsidian samples have not yet been completed, however the results of the studies carried out only in the two source areas belonging to the Nemrut volcano have been published by A.K. Robin et al. (2016).

\section{DEĞINIILEN BELGELER}

Arslan, M., 1994, Mineralogy, geochemistry, petrology and petrogenesis of the Meydan-Zilan (ErcişVan, Turkey) area volcanic rocks: Doktora Tezi, Glasgow Üniversitesi, 559s. (yayınlanmamış).

Astruc L., Gratuze B., Pelegrin J., Akkermans P., 2007. From production to use: a parcel of obsidian bladelets at Sabi Abyad II, in Astruc, Binder \& Briois (ed.), Systèmes techniques et communautés $\mathrm{du}$ néolithiques précéramique au Proche-Orient. Actes du 5e colloque international, Fréjus, du 29 février au 5 mars 2004, Antibes: APDCA, 327341.

Bigazzi G., Yegingil Z., Ercan T., Oddone M., Özdogan M., 1997. Age determination of obsidian bearing volcanics in eastern Anatolia using the Fissiontrack dating method. Geological Bulletin of Turkey, 40 (2): 57-72.

Bigazzi G., Poupeau G., Yegingil Z., Bellot-Gurlet L.,1998. Provenance studies of obsidian artefacts in Anatolia using the fission-track dating method: an overview. In M.-C. Cauvin et al., (ed). L'Obsidienne au Proche et Moyen Orient. BAR. Oxford: Archeopress: 69-89.

Binder, D., Gratuze, B., Mouralis, D., Balkan-Atl1, N., 2011. New investigations of the Göllüdağ obsidian lava flows system: a multidisciplinary approach. Journal of Archaeological Science. 38 (12), 3174-3184.

Blackman, J., R. Badaljan,Z.Kikodze, and P.Kohl.,1998. Chemical characterization of Caucasian obsidian geological sources. In M.-C. Cauvin et al., (ed) L'Obsidienne au Proche et Moyen Orient. BAR. Oxford: Archeopress: 205-231.

Cann, J.R. and C. Renfrew. 1964. The Characterization of Obsidian and Its Application to the Mediterranean Region. Proceedings of the Prehistoric Society. 30: 111-131.

Cauvin M. -C, Gourgaud A., Gratuze B., Arnaud N., Poupeau G., Poidevin J-L., 1998.Chataigner C., (ed). L'Obsidienne au Proche et Moyen Orient. BAR. Oxford: Archeopress, 388.

Cauvin, M.-C., Chataigner, C., 1998. Distribution de l'obsidienne dans les sites archéologiques du Proche et Moyen Orient. In: Cauvin, M.-C. (Ed.), L'obsidienne au Proche et Moyen Orient ancien: du volcan à l'outil, Lyon, Maison de l'Orient Méditerranéen 738. B.A.R Inter. Ser., Oxford, pp. 325-350. 
Chataigner C., Isikli M., Gratuze B. 2013. Obsidian sources in the regions of Erzurum and Kars (North-East Turkey): new data. Archaeometry. (DOI : 10.1111/arcm.12002). 1-24

Chataigner C., Gratuze B.. 2013a New data on the exploitation of the obsidian in the Southern Caucasus (Armenia, Georgia) and Eastern Turkey, Part 1 : Source characterization. Archaeometry.. (DOI :10.1111/arcm.12006).

Chataigner C., Gratuze B. 2013b. New data on the exploitation of the obsidian in the Southern Caucasus (Armenia, Georgia) and Eastern Turkey, Part 2 : Obsidian procurement from the Upper Palaeolithic to the Late Bronze Age. Archaeometry. (DOI:10.1111/arcm.12007).

Chataigner, C., 1994. Les propriétés géochimiques des obsidiennes et la distinction des sources de Bingöl et du Nemrut Dag. Paléorient 20 (2), 9-17.

Chataigner C, Poidevin J.L, Arnaud N., 1998. Turkish occurrences of obsidian and use by prehistoric peoples in the Near East from 14,000 to $6000 \mathrm{BP}$. Journal of Volcanology and Geothermal Research, 85 (1-4) (Octobre): 517-537.

Ercan, T., Şaroğlu, F., Kusçu, I., 1996. Features of obsidian beds formed by volcanic activity since 25 million years B.P. In: Dimirci, S., Özer, A.M., Summers, G.D. Eds. The Proceedings of the 29th International Symposium on Archaeometry, Ankara 1994. Tübitak, Ankara: 505-513.

Erturaç, K., Kuzucuoğlu, C., Mouralis, D., Astruc, L., Balkan-Atl1, N., 2010. The Göllüdağ complex: genesis of volcanoes and prehistoric societies. 1ères rencontres archéologiques de l'IFEA, Istanbul, 11-13 novembre 2010.

Frahm, E., 2012. Distinguishing Nemrut Dağ and Bingöl A obsidians: geochemical and landscape differences and the archeological implication. Journal of Archaeological Science. 39,1436-1444.
Gratuze, B.,1998. Les méthodes de caractérisation de l'obsidienne. In M.-C. Cauvin et al., (ed). L'Obsidienne au Proche et Moyen Orient. BAR. Oxford: Archeopress: 31-51.

Karaoğlu, Ö., Özdemir, Y., Tolluoğlu, A.Ü., Karabıyıkoğlu, M., Köse, O., Froger, J.-L., 2005. Stratigraphy of the volcanic products around Nemrut Caldera: implications for reconstruction of the caldera formation. Turk. Journal Earth Science. 14, 123-143.

Keller, J. et Seifried, C., 1990. The present status of obsidian source identification in Anatolia and the Near-East, PACT, 25: 57-87.

Khalidi, L., Gratuze B., Boucetta S., 2009. Provenance of obsidian excavated from late chalcolithic levels at the sites of Tell Hamoukar and Tell Brack. Archaeometry 51: 879-893.

Nomade, S., Gauthier, A., Guillou, H., J.F., P., 2010. 40Ar/39Ar temporal framework for the Alleret maar lacustrine sequence (French Massif-Central): volcanological and paleoclimatic implications. Quaternaire. Geochronology. 5, 20-27.

Oddone, M., Yeğinğil Z., Bigazzi G., Ercan T,. Özdoğan M., 1997. Chemical characterisations of Anatolian obsidians by instrumental and epithermal neutron activation analysis. Journal of Radioanalytical and Nuclear Chemistry. 224 (1-2) (octobre): 27-38.

Özdemir, Y., Karaoğlu, Ö., Tolluoğlu, A.U., Güleç, N., 2006. Volcanostratigraphy and petrogenesis of the Nemrut stratovolcano, East Anatolian High Plateau: the most recent post-collisional volcanism in Turkey. Chemical. Geology. 226, 189-211.

Özdoğan M., Başgelen N. 1999. Neolithic in Turkey. Arkeoloji ve Sanat Yayınları: 236.

Pearce J.A., Bender J.F., De Long S.E., Kidd W.S.F., Low P.J., Güner Y., Saroğlu F., Yilmaz Y., Moorbath S., Mitchell J.G. 1990. Genesis of collision volcanism in Eastern Anatolia, Turkey. 
Journal of Volcanology and Geothermal Research. 44 (1-2): 189-229.

Pearce J.A., Bender J.F., De Long S.E., Kidd W.S.F., Low P.J., Güner Y., Saroğlu F., Yilmaz Y., Moorbath S., Mitchell J.G. 1990. Genesis of collision volcanism in Eastern Anatolia, Turkey. Journal of Volcanology and Geothermal Research. 44 (1-2): 189-229.

Poidevin, J.L. 1998. Les gisements d'obsidienne de Turquie et de Transcaucasie: géologie, géochimie et chronométrie. In Cauvin et al. (ed.), L'Obsidienne au Proche et Moyen Orient. Du volcan à l'outil, BAR International Series 738. Oxford: Archeopress: 105-203.

Renfrew, C., Dixon, J.E., Cann, J.R., 1996. Obsidian and early cultural contact in the Near East. Proceedings of Prehistoric Society. 32: 30-72.

Robin, A.K., Mouralis, D., Akköprü, E., Gratuze B., , Kuzucuoğlu, C., Nomade, S., Pereira, A., Doğu, A.F., Erturaç, K., Khalidi, L., 2016. Identification and characterization of two new obsidian subsources in the Nemrut volcano (Eastern Anatolia, Turkey): The Sicaksu and Kayacık obsidian. Journal of Archaeological Science: Reports, Volume 9, October 2016, Pages 705-717
Yegingil, Z., Boztug, D., Er, M., Oddone, M., Bigazzi, G., 2002. Timing of neotectonic fracturing by fission track dating of obsidian in-filling faults in the Ikizdere-Rize area, NE Black Sea region, Turkey. Terra Nova. 14: 169-174.

Yılmaz, Y., Güner, Y., Şaroğlu, F., 1998. Geology of the Quaternary volcanic centres of the east Anatolia. J. Volcanol. Geotherm. Res. 85, 173-210.

$\begin{array}{ll}\text { Makale Geliş Tarihi } & : \text { 1 Eylül } 2016 \\ \text { Kabul Tarihi } & : 9 \text { Aralı } 2016 \\ \text { Received } & : \text { 1 September } 2016 \\ \text { Accepted } & : 9 \text { December } 2016\end{array}$


